\title{
On the Uncoded BER Performance Bound of the IEEE 802.16d Channel
}

\author{
Pei Xiao, Member, IEEE, Luis G. Barbero, Member, IEEE, Mathini Sellathurai, Senior Member, IEEE \\ T. Ratnarajah, Senior Member, IEEE
}

\begin{abstract}
The emergence of the IEEE 802.16 standard has spurred tremendous interest from operators seeking to deploy high-performance, cost-effective broadband wireless networks. It is an ideal solution for providing high data rate communications where traditional landlines are either unavailable or too costly to be installed. In this letter, the performance bound of the IEEE 802.16d channel is examined analytically in order to gain an insight into its theoretical potential. Different design strategies, such as orthogonal frequency division multiplexing (OFDM) and single-carrier frequency-domain equalization (SC-FDE), timedomain decision feedback equalization (DFE) and sphere decoder (SD) techniques are discussed and compared to the theoretical bound.
\end{abstract}

Index Terms-IEEE 802.16, performance bound, sphere decoder.

\section{INTRODUCTION}

Broadband wireless access has attracted considerable attention as a promising approach for the next generation high quality, high capacity and high density access infrastructure [1]. It is set to become a popular way to meet the escalating business demand for rapid Internet connection and integrated data, voice, and video services. The standardization activities have been performed under the auspices of the IEEE 802.16 working group, divided between $802.16 \mathrm{~d}$, i.e. fixed worldwide interoperability for microwave access (WiMAX), and 802.16e, i.e. mobile WiMAX [2]. In this work, we focus on the former case, and conduct a theoretical study of the IEEE 802.16d channel, obtaining a bit error rate (BER) performance bound that serves as a benchmark for comparison between different system implementations. Both orthogonal frequency division multiplexing (OFDM) and single-carrier solutions have been adopted in the IEEE 802.16d standard as possible alternatives for WiMAX systems operating in the 2-11 GHz band [3]. In this letter, we will present the results for both alternatives comparing their performances to the analytical bound derived in the sequel.

Tailored to different terrain conditions, a set of 6 typical wireless channel models called Stanford University Interim (SUI) channel models were proposed in [4], [5], used for simulation, design, development and testing of technologies suitable for WiMAX applications. All of them contain 3 taps, having either Rayleigh or Ricean amplitude distributions. For the purpose of this study, we assume an uncoded system with a data rate chosen such that the multipath fading is modelled as a tapped-delay line with adjacent taps equally spaced at the symbol rate. The received signal for a given sampling instant

The authors are with the Institute of Electronics, Communications and Information Technology (ECIT) at Queen's University of Belfast, BT3 9DT Belfast, UK (e-mail: pei.xiao@ecit.qub.ac.uk).

This work was supported by the UK Engineering and Physical Sciences Research Council under grant number EP/D07827X/1. $n$ can be written as

$$
r_{n}=h_{0} s_{n}+h_{1} s_{n-1}+h_{2} s_{n-2}+v_{n}
$$

where the channel coefficients $h_{0}, h_{1}, h_{2}$ are complex Gaussian distributed and assumed to remain constant during the transmission of one block of data. They, however, vary from block to block. The amplitude of the first tap $\left|h_{0}\right|$ is characterized by a Ricean distribution due to the line of sight propagation. The amplitudes of the taps $\left|h_{1}\right|,\left|h_{2}\right|$ are Rayleigh distributed. The transmitted symbol at sampling instant $n$ is denoted as $s_{n}$, and $v_{n}$ represents the complex additive white Gaussian noise with zero mean and variance $N_{0}$, i.e. $v_{n} \sim \mathcal{C N}\left(0, N_{0}\right)$.

Throughout this letter, $(\cdot)^{\mathrm{T}}$ denotes matrix transpose, $(\cdot)^{\mathrm{H}}$ matrix conjugate and $\mathrm{E}[\cdot]$ expectation operation.

\section{PERFORMANCE BOUND}

Based on (1), the received signal can be written in vector form, concatenating 3 sampling instants, as

$$
\underbrace{\left[\begin{array}{c}
r_{n} \\
r_{n+1} \\
r_{n+2}
\end{array}\right]}_{\mathbf{r}_{n}}=\underbrace{\left[\begin{array}{ccccc}
h_{2} & h_{1} & h_{0} & 0 & 0 \\
0 & h_{2} & h_{1} & h_{0} & 0 \\
0 & 0 & h_{2} & h_{1} & h_{0}
\end{array}\right]}_{\mathbf{H}} \underbrace{\left[\begin{array}{c}
s_{n-2} \\
s_{n-1} \\
s_{n} \\
s_{n+1} \\
s_{n+2}
\end{array}\right]}_{\mathbf{s}_{n}}+\underbrace{\left[\begin{array}{c}
v_{n} \\
v_{n+1} \\
v_{n+2}
\end{array}\right]}_{\mathbf{v}_{n}} .
$$

Suppose $s_{n}$ is the symbol of interest. Assuming perfect knowledge of $\mathbf{H}$, and defining $\hat{\mathbf{s}}_{n}=$ $\left[\begin{array}{lllll}\hat{s}_{n-2} & \hat{s}_{n-1} & 0 & \hat{s}_{n+1} & \hat{s}_{n+2}\end{array}\right]^{\mathrm{T}}$ as the vector containing the estimate of the interference symbols, the interference cancelled signal vector is given by

$$
\mathbf{r}_{n}^{\prime}=\mathbf{r}_{n}-\mathbf{H} \hat{\mathbf{s}}_{n}=\mathbf{H}\left(\mathbf{s}_{n}-\hat{\mathbf{s}}_{n}\right)+\mathbf{v}_{n},
$$

which can be rewritten as

$$
\mathbf{r}_{n}^{\prime}=\left[\begin{array}{c}
r_{n}^{\prime} \\
r_{n+1}^{\prime} \\
r_{n+2}^{\prime}
\end{array}\right]=\left[\begin{array}{c}
h_{0} \\
h_{1} \\
h_{2}
\end{array}\right] s_{n}+\left[\begin{array}{c}
w_{n} \\
w_{n+1} \\
w_{n+2}
\end{array}\right]=\mathbf{h} s_{n}+\mathbf{w}_{n}
$$

where $\mathbf{h}=\left[\begin{array}{lll}h_{0} & h_{1} & h_{2}\end{array}\right]^{\mathrm{T}}$ represents the channel vector and $\mathbf{w}_{n}$ denotes the combined noise and interference cancellation residual vectors.

In order to obtain a performance bound for the above system, we assume perfect cancellation in (3). In this case, all the cancellation residuals will vanish, i.e. $\mathbf{w}_{n}=\mathbf{v}_{n}$. For maximum exploitation of the multipath diversity gain, the decision statistic for the symbol $s_{n}$ can be derived by applying maximum ratio combining [6], i.e.

$$
z_{n}=\mathbf{h}^{\mathrm{H}} \mathbf{r}_{n}^{\prime} / \mathcal{P}=s_{n}+\eta_{n}
$$


where $\mathcal{P}=\sum_{i=0}^{2}\left|h_{i}\right|^{2}$, and $\eta_{n} \sim \mathcal{C N}\left(0, N_{0} / \mathcal{P}\right)$. The symbol $s_{n}$ can then be estimated by making a hard decision on $z_{n}$ using a maximum likelihood (ML) decision rule. For the QPSK signal constellation, the bit error probability can be computed according to [7] as

$$
P_{b}=Q\left(\frac{\sqrt{E_{b} \sum_{l=0}^{2}\left|h_{l}\right|^{2}}}{\sqrt{N_{0} / 2}}\right)=Q\left(\sqrt{\frac{2 E_{b} \mathcal{P}}{N_{0}}}\right)
$$

where $Q(x)=\int_{x}^{\infty} \frac{1}{\sqrt{2 \pi}} \exp \left(-t^{2} / 2\right) d t$ is the complementary Gaussian cumulative distribution function. Denoting $x=\left|h_{0}\right|^{2}$ and $y=\left|h_{1}\right|^{2}+\left|h_{2}\right|^{2}$, the bit error probability can be written as a function of the random variables $x$ and $y$, i.e.

$$
P_{b}(x, y)=Q\left(\sqrt{\frac{2 E_{b}(x+y)}{N_{0}}}\right)
$$

Since $\left|h_{0}\right|$, the amplitude of the first tap, is Ricean distributed due to the existence of line of sight propagation, the random variable $x$ is non-central Chi-square distributed with 2 degrees of freedom and probability density function (pdf)

$$
p(x)=\frac{1}{2 \sigma^{2}} \exp \left(-\frac{x+s^{2}}{2 \sigma^{2}}\right) I_{0}\left(\frac{\sqrt{x} s}{\sigma^{2}}\right),
$$

where $x \geq 0, I_{0}(x)$ is the $0^{t h}$ order modified Bessel function of the first kind [8, p. 44]; $s^{2}=m_{r}^{2}+m_{i}^{2}$, and $m_{r}, m_{i}, \sigma^{2}$ stand for the mean value and variance of the real and imaginary part of $h_{0}$, respectively. The amplitudes of the other two taps, $\left|h_{1}\right|$ and $\left|h_{2}\right|$, are characterized by a Rayleigh distribution. Therefore, the random variables $\left|h_{1}\right|^{2}$ and $\left|h_{2}\right|^{2}$ have a central Chisquare distribution with 2 degrees of freedom and characteristic functions

$$
\psi_{\left|h_{1}\right|^{2}}(j v)=\left(1-j v \gamma_{1}\right)^{-1} ; \quad \psi_{\left|h_{2}\right|^{2}}(j v)=\left(1-j v \gamma_{2}\right)^{-1},
$$

where $\gamma_{1}=\mathrm{E}\left[\left|h_{1}\right|^{2}\right], \gamma_{2}=\mathrm{E}\left[\left|h_{2}\right|^{2}\right]$, and $\gamma_{1} \neq \gamma_{2}$. As a consequence of the statistical independence of $\left|h_{1}\right|^{2}$ and $\left|h_{2}\right|^{2}$, the characteristic function of $y$ is

$$
\begin{aligned}
& \psi_{y}(j v)=\left(1-j v \gamma_{1}\right)^{-1}\left(1-j v \gamma_{2}\right)^{-1} \\
& =\left(1-\frac{\gamma_{2}}{\gamma_{1}}\right)^{-1}\left(1-j v \gamma_{1}\right)^{-1}+\left(1-\frac{\gamma_{1}}{\gamma_{2}}\right)^{-1}\left(1-j v \gamma_{2}\right)^{-1} \\
& =\frac{\gamma_{1}}{\gamma_{1}-\gamma_{2}}\left(1-j v \gamma_{1}\right)^{-1}+\frac{\gamma_{2}}{\gamma_{2}-\gamma_{1}}\left(1-j v \gamma_{2}\right)^{-1}
\end{aligned}
$$

Taking its inverse Fourier transform, we obtain the pdf of $y$ as

$$
p(y)=\frac{1}{\gamma_{1}-\gamma_{2}} \exp \left(-\frac{y}{\gamma_{1}}\right)+\frac{1}{\gamma_{2}-\gamma_{1}} \exp \left(-\frac{y}{\gamma_{2}}\right),
$$

where $y \geq 0$. To obtain the average bit error probability, we must average $P_{b}(x, y)$ in (4) over the random variables $x$ and $y$, i.e. the average BER can be calculated as

$$
\begin{aligned}
\bar{P}_{b} & =\int_{0}^{\infty} \int_{0}^{\infty} P_{b}(x, y) p(x) p(y) d x d y \\
& =\int_{0}^{\infty} \int_{0}^{\infty} Q\left(\sqrt{\frac{2 E_{b}(x+y)}{N_{0}}}\right) p(x) p(y) d x d y
\end{aligned}
$$

where $p(x)$ and $p(y)$ are defined in (5) and (6), respectively.
The expression in (7) can be evaluated numerically but it can also be derived analytically. Next, we provide a closedform expression of (7), in order to avoid numerical integration. Using the inequality [9, p. 100]

$$
Q(\sqrt{\alpha+\beta}) \leq Q(\sqrt{\alpha}) \exp (-\beta / 2)
$$

for $\alpha, \beta \geq 0$, we can obtain an approximation to (7), in the form of an upper-bound, as a product of two terms

$$
\bar{P}_{b} \leq \underbrace{\int_{0}^{\infty} Q\left(\sqrt{\frac{2 E_{b} x}{N_{0}}}\right) p(x) d x}_{\Phi} \cdot \underbrace{\int_{0}^{\infty} \exp \left(-\frac{E_{b} y}{N_{0}}\right) p(y) d y}_{\Psi}
$$

where

$$
\begin{aligned}
\Phi= & \frac{\exp \left(-s^{2} / 2 \sigma^{2}\right)}{2 \sigma^{2}} \\
& \cdot \int_{0}^{\infty} Q\left(\sqrt{\frac{2 E_{b} x}{N_{0}}}\right) \exp \left(-\frac{x}{2 \sigma^{2}}\right) I_{0}\left(\frac{\sqrt{x} s}{\sigma^{2}}\right) d x
\end{aligned}
$$

and

$$
\begin{aligned}
\Psi= & \frac{1}{\gamma_{1}-\gamma_{2}} \int_{0}^{\infty} \exp \left(-\frac{E_{b} y}{N_{0}}\right) \exp \left(-\frac{y}{\gamma_{1}}\right) d y \\
& +\frac{1}{\gamma_{2}-\gamma_{1}} \int_{0}^{\infty} \exp \left(-\frac{E_{b} y}{N_{0}}\right) \exp \left(-\frac{y}{\gamma_{2}}\right) d y
\end{aligned}
$$

The integral in (9) can be solved by using the following equation [10]:

$$
\begin{aligned}
\int_{0}^{\infty} Q(\sqrt{a x}) x^{L-1} \exp (-x / b) d x & \\
= & \frac{1}{2} b^{L} \Gamma(L)\left[1-\sum_{k=0}^{L-1} \mu\left(\frac{1-\mu^{2}}{4}\right)^{k}\left(\begin{array}{c}
2 k \\
k
\end{array}\right)\right],
\end{aligned}
$$

where $\mu=\sqrt{\frac{a b}{2+a b}}$ and $\Gamma(x)=\int_{0}^{\infty} t^{x-1} \exp (-t) d t$ is the Gamma function. We then apply the above result to (9), denoting $a=2 E_{b} / N_{0}, b=2 \sigma^{2}$, to obtain

$$
\begin{aligned}
& \int_{0}^{\infty} Q\left(\sqrt{\frac{2 E_{b} x}{N_{0}}}\right) \exp \left(-\frac{x}{2 \sigma^{2}}\right) I_{0}\left(\frac{\sqrt{x} s}{\sigma^{2}}\right) d x \\
& =\int_{0}^{\infty} Q\left(\sqrt{\frac{2 E_{b} x}{N_{0}}}\right) \exp \left(-\frac{x}{2 \sigma^{2}}\right) \sum_{l=0}^{\infty} \frac{x^{l} s^{2 l}}{\left(2 \sigma^{2}\right)^{2 l}(l !)^{2}} d x \\
& =\frac{1}{2} \sum_{l=0}^{\infty}\left(2 \sigma^{2}\right)^{l+1} \Gamma(l+1) \\
& \cdot\left[1-\sum_{k=0}^{l} \mu\left(\frac{1-\mu^{2}}{4}\right)^{k}\left(\begin{array}{c}
2 k \\
k
\end{array}\right)\right] \frac{s^{2 l}}{\left(2 \sigma^{2}\right)^{2 l}(l !)^{2}} \\
& =\frac{1}{2} \sum_{l=0}^{\infty}\left[1-\sum_{k=0}^{l} \mu\left(\frac{1-\mu^{2}}{4}\right)^{k}\left(\begin{array}{c}
2 k \\
k
\end{array}\right)\right] \frac{s^{2 l}}{\left(2 \sigma^{2}\right)^{l-1} l !}
\end{aligned}
$$

Thus, substituting (12) into (9) yields

$$
\begin{aligned}
\Phi= & \frac{\exp \left(-s^{2} / 2 \sigma^{2}\right)}{4 \sigma^{2}} \\
& \cdot \sum_{l=0}^{\infty}\left[1-\sum_{k=0}^{l} \mu\left(\frac{1-\mu^{2}}{4}\right)^{k}\left(\begin{array}{c}
2 k \\
k
\end{array}\right)\right] \frac{s^{2 l}}{\left(2 \sigma^{2}\right)^{l-1} l !} .
\end{aligned}
$$


Meanwhile, the computation of $\Psi$ in (10) results in

$$
\begin{aligned}
\Psi & =\frac{1}{u_{1}\left(\gamma_{1}-\gamma_{2}\right)}+\frac{1}{u_{2}\left(\gamma_{2}-\gamma_{1}\right)} \\
& =\frac{N_{0} \gamma_{1}}{\left(\gamma_{1}-\gamma_{2}\right)\left(E_{b} \gamma_{1}+N_{0}\right)}+\frac{N_{0} \gamma_{2}}{\left(\gamma_{2}-\gamma_{1}\right)\left(E_{b} \gamma_{2}+N_{0}\right)},
\end{aligned}
$$

where

$$
u_{1}=\frac{E_{b}}{N_{0}}+\frac{1}{\gamma_{1}}=\frac{E_{b} \gamma_{1}+N_{0}}{N_{0} \gamma_{1}} ; \quad u_{2}=\frac{E_{b} \gamma_{2}+N_{0}}{N_{0} \gamma_{2}} .
$$

Combining (8), (13) and (14), we obtain the final closedform expression of the BER performance bound of the IEEE $802.16 \mathrm{~d}$ channel as

$$
\begin{aligned}
\bar{P}_{b} \leq & \frac{\exp \left(-s^{2} / 2 \sigma^{2}\right)}{4 \sigma^{2}} \\
& \cdot \sum_{l=0}^{\infty}\left[1-\sum_{k=0}^{l} \mu\left(\frac{1-\mu^{2}}{4}\right)^{k}\left(\begin{array}{c}
2 k \\
k
\end{array}\right)\right] \frac{s^{2 l}}{\left(2 \sigma^{2}\right)^{l-1} l !} \\
& \cdot\left[\frac{N_{0} \gamma_{1}}{\left(\gamma_{1}-\gamma_{2}\right)\left(E_{b} \gamma_{1}+N_{0}\right)}+\frac{N_{0} \gamma_{2}}{\left(\gamma_{2}-\gamma_{1}\right)\left(E_{b} \gamma_{2}+N_{0}\right)}\right] \\
= & \frac{\exp \left(-s^{2} / 2 \sigma^{2}\right) N_{0}}{4 \sigma^{2}\left(\gamma_{1}-\gamma_{2}\right)}\left[\frac{\gamma_{1}}{E_{b} \gamma_{1}+N_{0}}-\frac{\gamma_{2}}{E_{b} \gamma_{2}+N_{0}}\right] \\
& \cdot \sum_{l=0}^{\infty}\left[1-\sum_{k=0}^{l} \mu\left(\frac{1-\mu^{2}}{4}\right)^{k}\left(\begin{array}{c}
2 k \\
k
\end{array}\right)\right] \frac{s^{2 l}}{\left(2 \sigma^{2}\right)^{l-1} l !},
\end{aligned}
$$

which can be used to assess how the performance of actual uncoded systems compares to the best achievable uncoded performance in the IEEE $802.16 \mathrm{~d}$ channel.

\section{ANALyticAl AND SIMUlation RESUlts}

For the purpose of this study, we select the SUI-2 and SUI-3 channels, which correspond to average British suburban conditions. The parameters for each channel model are chosen according to [5] and are summarized in Table I. We use quadrature phase-shift keying (QPSK) modulation, and the data rate is chosen to be $4 \mathrm{Mbp}$ so that the symbol duration equals the channel tap spacing, which is $0.5 \mu \mathrm{s}$. We compare the theoretical BER performance bounds obtained in Section II to the performance of both frequency-domain and time-domain based systems, measured through Monte Carlo simulations.

For the frequency-domain case, the following two schemes are considered: orthogonal frequency division multiplexing (OFDM) as introduced in [14], and single-carrier frequencydomain equalization (SC-FDE) as in [15], [16]. The main difference between SC-DFE and OFDM is that the inverse fast Fourier transform (FFT) block is moved to the receiver to convert frequency-domain equalized signals back into timedomain symbols. For channels with severe delay spread, it is simpler than the corresponding time domain equalization due to the use of the computationally efficient FFT operation [15]. In both cases, each frame of data contains 4,096 information bits (2,048 QPSK symbols), divided into 32 OFDM blocks. The number of subcarriers is set to 64 and 8 samples are used for the cyclic prefix. A minimum mean-square error (MMSE) detector is used for symbol estimation at the receiver [15].

For the time-domain case, two schemes are also considered.

\begin{tabular}{|c|c|c|}
\hline & SUI-2 channel & SUI-3 channel \\
\hline Tap power $(\mathrm{dB})$ & {$\left[\begin{array}{lll}0 & -12 & -15\end{array}\right]$} & {$\left[\begin{array}{lll}0 & -5 & -10\end{array}\right]$} \\
\hline $\mathrm{K}$ factor & {$\left[\begin{array}{lll}2 & 0 & 0\end{array}\right]$} & {$\left[\begin{array}{lll}1 & 0 & 0\end{array}\right]$} \\
\hline$s^{2}, \sigma^{2}$ & $0.6, \quad 0.153$ & $0.36, \quad 0.175$ \\
\hline$\gamma_{1}, \gamma_{2}$ & $0.057, \quad 0.029$ & $0.223, \quad 0.07$ \\
\hline
\end{tabular}
Firstly, we use decision-feedback equalization (DFE) as presented in [8]. The equalizer has 5 feedforward symbol-spaced taps and 3 feedback symbol-spaced taps. The block size is set to 10,000 bits, corresponding to 5,000 QPSK symbols. The first 200 symbols of each transmitted block are used as pilot

\section{TABLE I}

PARAMETER SETTINGS FOR SUI-2,-3 CHANNELS.

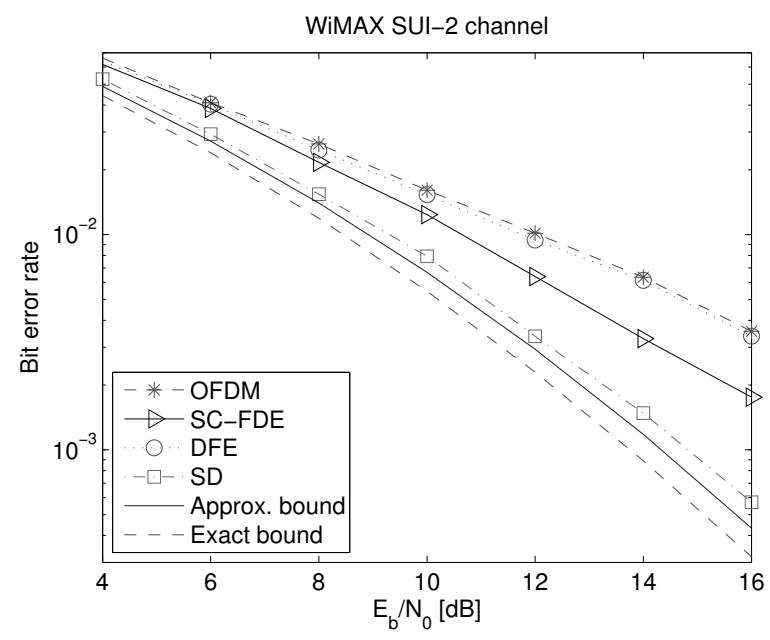

Fig. 1. BER performance bound and BER of time-domain and frequencydomain systems in a SUI-2 channel using QPSK modulation.

symbols to train the equalizer coefficients, using the recursive least-squares (RLS) method presented in [11]. Secondly, we use the sphere decoder (SD) as applied to frequency-selective channels in [12]. The SD performs a depth-first metricconstrained tree search on a triangular decomposition of the channel matrix [13]. In contrast to [12], the SD simulated here makes use of the Schnorr-Euchner enumeration [13], further reducing the complexity of the original SD. The initial radius in the SD is set initially to $\infty$ and reduced every time the tree search obtains a full-length path satisfying the metric constraint. Due to the joint detection performed in this case, the block size is set to 20 QPSK symbols, which does not change the achievable performance of the SD.

Fig. 1 and Fig. 2 show the BER performance of the different schemes compared to the analytical BER performance bounds in the SUI-2 and SUI-3 channels, respectively, as a function of $E_{b} / N_{0}=1 /\left(2 N_{0}\right)$, which denotes the signal to noise ratio (SNR) per bit when the average symbol energy is set to unity. The approximate bound is given by (15); the exact bound is derived by numerical evaluation of (7). It can be observed that, in both cases, the approximate bound is a tight bound, differing from the exact bound by a maximum of 0.4-0.5 $\mathrm{dB}$ for both channels. By comparison, the SUI-3 channel has a lower bound than the SUI-2 channel. This follows from the fact that the SUI-2 channel has a more dominant line-of-sight component as shown in Table I, and the non-line-of-sight taps are much weaker than the ones in the SUI-3 channel, leading to less multipath diversity.

As expected, the performance of SC-FDE is better than that of OFDM. In order to exploit diversity in an OFDM system, channel coding has to be applied. It was shown in [17] that OFDM outperforms SC-DFE in coded WiMAX systems. It can also be seen that OFDM yields comparable and inferior 


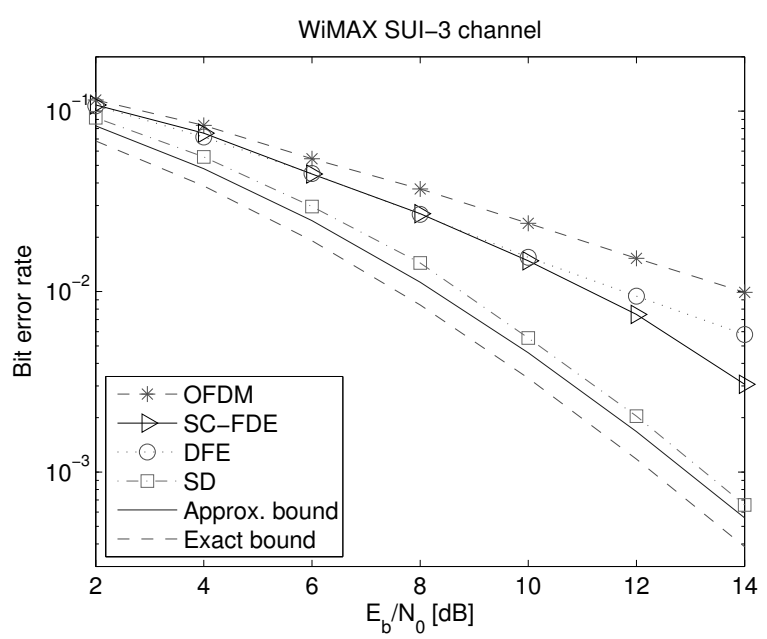

Fig. 2. BER performance bound and BER of time-domain and frequencydomain systems in a SUI-3 channel using QPSK modulation.

performance to DFE in the SUI-2 and the SUI-3 channels, respectively. Since OFDM transforms a frequency selective fading channel into a flat fading channel, the multipath signals become non-resolvable, forgoing the opportunity to make use of the effective multipath diversity gain, especially in the SUI-3 channel. On the contrary, DFE exploits the temporal diversity obtained from multipath propagation. However, compared to OFDM, the gain obtained by DFE is much smaller in the SUI-2 channel due to the reduced multipath diversity.

In both channels, SC-FDE performs better than DFE and OFDM, although it is still far above the performance bounds (2-4 dB away from the exact and approximate bounds at BERs between $10^{-2}$ and $10^{-3}$ ). Finally, it can be observed that the SD outperforms all the other schemes and approaches the BER performance bounds obtained in this letter, achieving the same diversity gain with a gap of $0.2-0.4 \mathrm{~dB}$ and less than $1 \mathrm{~dB}$ from the approximate bound and the exact bound, respectively. This is due to the joint detection that is performed over the entire block, resulting in a near-ML performance. However, the main disadvantage of the SD is its potentially high complexity compared to the other schemes. Although it is lower than that of the MLD, and lower than that of the Viterbi algorithm at high SNR [12], it is still exponential for large block sizes and high constellation orders [19], which explains why the BER performance has been obtained for a relatively small block size compared to the other receivers under study. The complexity of the SD can be reduced by performing the tree search in different stages as presented for multiple-antenna systems in [20]. This solution would cause a small performance degradation with no apparent diversity loss. Furthermore, in order for the SD to be considered for practical systems in frequency-selective environments, the problem of its variable complexity would also need to be addressed [19].

\section{CONCLUSiOnS}

In this letter, we have derived an analytical bound, in closedform, on the BER performance of the IEEE 802.16d channel. Different transceiver schemes such as time-domain DFE and $\mathrm{SD}$, and frequency-domain OFDM and SC-FDE have been discussed with their performance compared to the theoretical bound. Our study reveals that the performance bound cannot be approached by conventional techniques, such as DFE, OFDM or SC-FDE. The solution in the current standard is to employ channel coding in conjunction with these techniques. However, the performance improvement comes at the expense of a reduced transmission rate and spectrum efficiency together with an increased receiver complexity. Results from this work also show that the ML solution obtained by the SD approaches the performance bound with no diversity loss, enabling an uncoded system to achieve an improved performance without having to resort to channel coding, sacrificing the data rate. Inspired by this finding, the design of a practical sphere decoding scheme with affordable complexity is currently being undertaken by the authors. Evolution of WiMAX systems will continue for many years to come and we believe that the results from this work could provide a useful reference for future versions of the IEEE802.16 standard.

\section{REFERENCES}

[1] H. Bolcskei, A. Paulraj, K. Hari, R. Nabar, W. Lu. "Fixed broadband wireless access: state of the art, challenges, and future directions". IEEE Commun. Mag., pp. 100-108, Jan. 2001.

[2] IEEE 802.16 Working Group on Broadband Wireless Access Standards. Available at http://grouperieee.org/groups/802/16/.

[3] C. Eklund. "IEEE Standard 802.16: a technical overview of the wirelessMAN air interface for broadband wireless access". IEEE Commun. Mag., pp. 98-107, June, 2002.

[4] V. Erceg. "An empirically based path loss model for wireless channels in suburban environments". IEEE J. Sel. Areas Commun., vol. 17, no. 7, pp. 1205-1211, July 1999.

[5] V. Erceg et al. "Channel models for fixed wireless applications". IEEE 802.16a cont. IEEE 802.16.3c-01/29r4, June 2003.

[6] D. Tse and P. Viswanath, Fundamentals of Wireless Communication. Cambridge University Press, 2005.

[7] P. Xiao, R. Carrasco, I. Wassell. "Performance analysis of conventional detection in BFWA systems". In Proc. Second IFIP International Conference on Wireless and Optical Communications Networks, (WOCN '05), Dubai, UAE, Mar. 2005, pp. 447-452.

[8] J. Proakis. Digital Communications, 4th edition, McGraw-Hill, 2000.

[9] S. Verdu. Multiuser Detection, 1st edition, Cambridge University Press, 1998.

[10] H. Zhang, T. Gulliver. "Error probability for maximum ratio combining multichannel reception of M-ary coherent systems over flat ricean fading channels". In Proc. IEEE Wireless Communications and Networking Conference (WCNC '04), vol. 1, Atlanta, GA, USA, Mar. 2004, pp. 306310.

[11] S. Haykin. Adaptive Filter Theory, 4th edition, Prentice Hall, 2002.

[12] H. Vikalo, B. Hassibi, U. Mitra. "Sphere-constrained ML detection for frequency-selective channels". IEEE Trans. Commun., vol. 54, no. 7, pp. 1179-1183, July 2006.

[13] A. D. Murugan, H. E. Gamal, M. O. Damen, and G. Caire, "A unified framework for tree search decoding: rediscovering the sequential decoder". IEEE Trans. on Inform. Theory, vol. 52, no. 3, pp. 933- 953, Mar. 2006.

[14] S. Weinstein, P. Ebert. "Data transmission by frequency-division multiplexing using discrete Fourier transform". IEEE Trans. Commun. Tech., vol. 19 , no. 5 , pp. 628-634, Oct. 1971.

[15] H. Sari, G. Karam, I. Jeanclaude. "Transmission techniques for digital terrestrial TV broadcasting". IEEE Commun. Mag., vol. 33, no. 2, pp. 100-109, Feb. 1995.

[16] D. Falconer, S. Ariyavisitakul, A. Benyamin-Seeyar, B. Eidson. "Frequency domain equalization for single-carrier broadband wireless systems". IEEE Commun. Mag., vol. 40, no. 4, pp. 58-66, April 2002.

[17] I. Chatzigeorgiou, M. Rodrigues, I. Wassell, R. Carrasco. "Turbo coded OFDM/SC-FDE techniques for MIMO BFWA channels". In Proc. First International Symposium on Broadband Communications (ISBC '04), Harrogate, UK, Dec. 2004.

[18] W. Webb. "Broadband fixed wireless access as a key component of the future integrated communications environment". IEEE Commun. Mag., vol. 39, no. 9, pp. 115-121, Sep. 2001.

[19] J. Jaldén and B. Ottersten, "On the complexity of sphere decoding in digital communications". IEEE Trans. on Sig. Proc., vol. 53, no. 4, pp. 1474-1484, Apr. 2005.

[20] L. G. Barbero, T. Ratnarajah, and C. Cowan, "A multistage sphere decoder for frequency-selective MIMO channels". In Proc. IEEE International Conference on Personal, Indoor and Mobile Radio Communications (PIMRC '07), Athens, Greece, Sep. 2007. 